\title{
A Propagation Modeling for Microcellular Communications in Urban Environments with Vehicles and Trees
}

\author{
Shiun-Chi Jan and Shyh-Kang Jeng \\ Department of Electrical Engineering, National Taiwan University, \\ Taipei 10617, Taiwan, R.O.C.
}

\section{Introduction}

Buildings, vehicles, and trees affect the radio wave propagation in a microcell for mobile radio communications. There have been a lot of papers discussing the propagation loss, cell coverage, and delay spread in simple environment using ray tracing technique and multiple-image concept [1][2]. Although these methods were useful, in their formulations the streets were empty - no cars, no trucks, and no trees. In this paper, we combine 2D ray tracing and simple $3 \mathrm{D}$ geometric considerations to generate an efficient and comprehensive three dimensional propagation model for a general propagation environment as shown in Fig. 1. By this approach, we can handle any distribution of buildings, vehicles, and trees in an urban environment. To the authors' knowledge, few papers considered the effects of vehicles. One of them examined the effect of vehicles in the tunnel [3] and another reported the measurement of signal attenuation caused by a large vehicle [4].

\section{Propagation Modeling}

A typical urban environment consists of streets, buildings, vehicles and groups of trees on safeisland. The streets are lined with tall buildings. The vehicles and groups of trees on safeisland are modeled by perfect electric conductor boxes and dielectric slabs, respectively. Figure 1 shows a plane view of the street scene. Consider the projection Txp of a transmitting antenna $T x$ on ground. We shoot 360 or $36002 \mathrm{D}$ ray tubes from $T x p$ and trace each ray tube which may bounce from the projections of the buildings, vehicles, and trees or transmit through the projections of trees and vehicles as shown in Fig. 4. A ray tube hits the projection of a vehicle or a group of trees is split into a reflected and a transmitted ray tube. Based on this idea, a binary reflection/transmission tree for a ray tube shot from $T x p$ is constructed. For each ray tube, we check whether the projection $R x p$ of the receiver $R x$ falls within the ray tube. If it does, an exact $2 \mathrm{D}$ ray from $T x p$ to $R x p$ can be determined using the 2D image sources. If $R x p$ doesn't fall within the ray tube, keep on tracing until all the branches are traversed.

Once an exact $2 \mathrm{D}$ ray from $T x p$ to $R x p$ is established, the third dimension is taken into consideration to determine if an exact $3 \mathrm{D}$ ray from $T x$ to $R x$ exists. Let there be $\mathrm{m}$ reflections and $\mathrm{n}$ transmissions for an exact $2 \mathrm{D}$ ray. If all the heights of the $m$ reflection points are lower than those of the $\mathrm{m}$ obstacles, an exact $3 \mathrm{D}$ ray exists. Otherwise the ray can not reach the receiver. Then we check whether the ray transmit through the $n$ trees or vehicles or fly over the top of them to the receiver. If the height of the transmission point is higher than that of the obstacle, the ray flies over the top of the obstacle. If not, the ray penetrates the obstacle when it is a group of trees, or is blocked and thus ignored when the obstacle is a vehicle. 
Then we compute the received power at the receiver. If the $3 \mathrm{D}$ ray is with reflections from $m$ obstacles and transmission through $\mathrm{n}$ obstacles, the field at the receiver is given by

$$
\vec{E}^{r}=\vec{E}^{i} \cdot \prod_{i=1}^{m} \overline{\overline{R_{i}}} \cdot \prod_{j=1}^{n} \overline{\overline{T_{j}}} \cdot e^{-\jmath(k j-k) d_{j}} \cdot \frac{e^{-\jmath k s}}{s}
$$

where $\bar{E}^{i}$ is the electric field at $\operatorname{Im}$ from the transmitter; $\overline{\overline{R_{i}}}$ is the dyadic reflection coefficient at the i'th reflection point; $\overline{\overline{T_{j}}}$ is the dyadic transmission coefficient at the $j$ 'th transmission point; $k_{j}$ is the wave number in the $j$ 'th group of trees; $d_{j}$ is the ray path length in the $j$ 'th group of trees; $s$ is the ray path length from the transmitter to the receiver; $k$ is the wave number in free space. Repeat tracing the ray tubes until all ray tubes have been traced and their contributions have been included, then we can get the propagation loss.

\section{Results}

Simulations were arranged to determine the propagation loss in a straight street with various distributions of vehicles and trees. The street width was $20 \mathrm{~m}$. The relative dielectric constant and absolute conductivity of the buildings and ground were 4.0 and $0.00075 \mathrm{~S} / \mathrm{m}[5]$, respectively. We computed the propagation loss for radio wave of $1 \mathrm{GHz}$. A Truck on the road was modeled by a PEC box with a length of $5.6896 \mathrm{~m}$, a width of $2.4384 \mathrm{~m}$, and a height of $4.6736 \mathrm{~m}$. The groups of trees on safeisland were modeled by a $80 \mathrm{~m} \times 1 \mathrm{~m} \times 4 \mathrm{~m}$ dielectric slab of which relative dielectric constant and absolute conductivity were 1.2 and $0.0001 \mathrm{~S} / \mathrm{m}[5]$, respectively. For the cases given in this paper, the $\mathrm{x}$-axis was set to be along the street. The transmitter placed at $\mathrm{x}=0$ was established at a height $h_{t}=6 \mathrm{~m}$, while the receiver moved at a fixed height $h_{r}=1.5 \mathrm{~m}$.

To get the effect of trucks, we randomly placed ten trucks in the $50 \mathrm{~m}$ long street region. For each case, we could find the severe fast fading and blockage due to reflection and shadowing by vehicles, respectively. The mean propagation losses of 8 cases are shown in Fig. 3. We can find that the received power is lower than the no-truck case, and the difference increases with the distance. Figure 4 shows the propagation loss due to groups of trees on safeisland. From the results, we found the attenuation due to groups of trees on safeisland were more than $10 \mathrm{~dB}$. The attenuation was even more obvious in the farther region.

\section{Conclusion}

The hybrid method combined with $2 \mathrm{D}$ ray tracing and simple $3 \mathrm{D}$ geometry considerations has been generated an efficient and comprehensive propagation modeling for a general propagation environment with buildings, vehicles, and trees. We have investigated the effects of vehicles and groups of trees on safeisland. Attenuation due to shadowing from vehicle and transmission from groups of trees are significant. More simulation results will be presented in the conference.

Acknowledgment This work was supported by the Telecommunication Laboratory, Directorate General of Telecommunications, under Grant TL-842205. 


\section{References}

[1 ] V. Erceg, S. Ghassemzadeh, M. Talor, D. Li, and P. L. Schilling, "Urban/suburban out-of-sight propagation modeling," IEEE Commun. Mag., vol. 30, no. 6, pp. 56-61, June 1992.

[2 ] S. Y. Tan and H. S. Tan, "A theory for propagation path-loss characteristics in a city-street grid," IEEE Trans. Electromagn. Compat., vol. EMC-37, no. 3, pp. 333-342, Aug. 1995.

[3 ] Y. Yamaguchi, T. Abe and T. Sekiguchi, "Radio wave propagation loss in the VHF to microwave region due to vehicles in tunnels," IEEE Trans. Electromagn. Compat., vol. EMC-31, no. 1, pp. 87-91, Feb. 1989.

[4 ] A. J. Rustako Jr., M. J. Gan, G. J. Owens and R. S. Roman, "Attenuation and diffraction effects from truck blockage of an 11-GHz line-of-sight microcellular mobile radio path," IEEE Trans. Veh. Technol., vol. VT40, no. 1, pp. 211-215, Feb. 1991.

[5 ] M. Lebherz, W. Wiesbeck, and W. Krank, "A versatile wave propagation model for the VHF/UHF range considering three-dimensional terrain," IEEE Trans. Antennas Propagat., vol. AP-40, pp. 1121-1131, Oct. 1992.
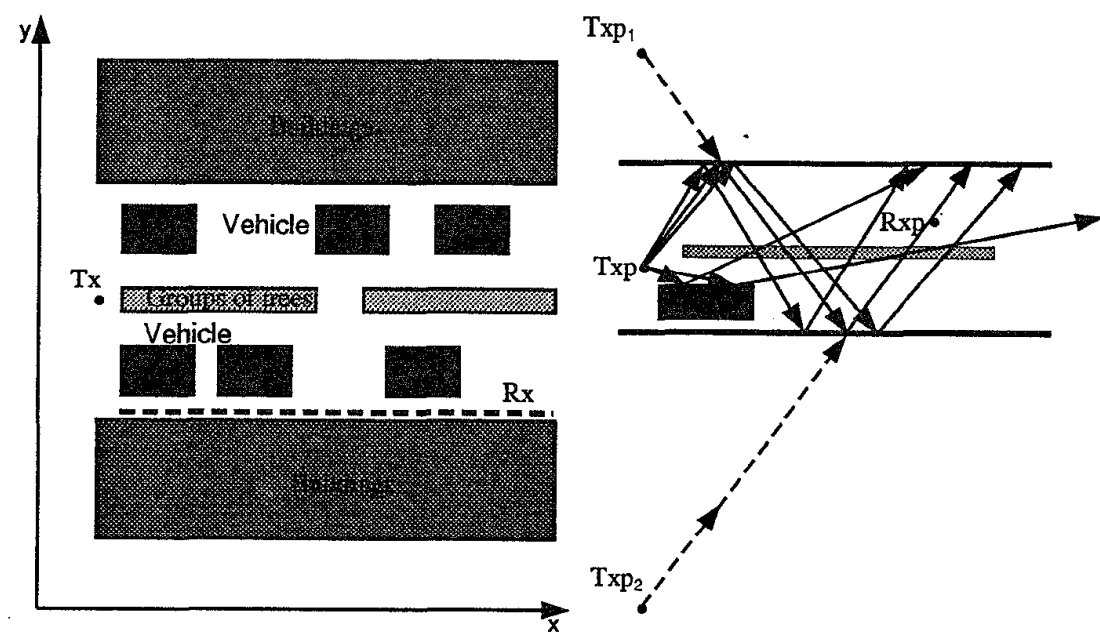

Fig. 1Plane view of a typical street scene.

Fig. 2 Geometry of 2D ray tracing. Txp and Rxp are the projections of transmitting antenna and receiving antenna on ground respectively. Txp1 and Txp2 are the 2D images of the first and second bounces. 


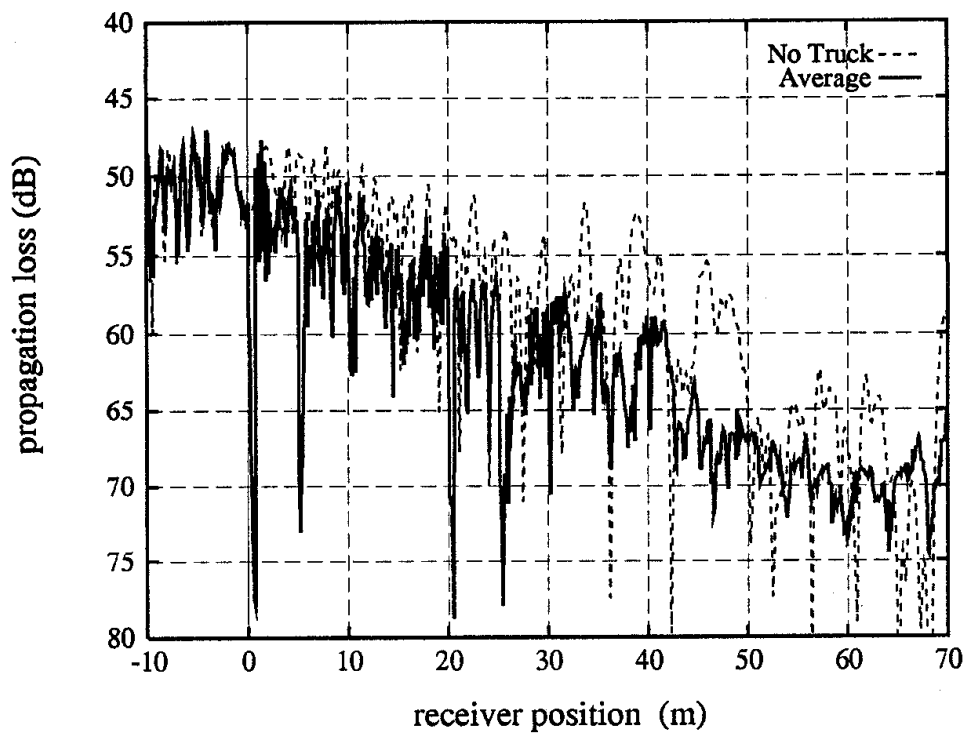

Fig. 3 Mean propagation loss variation according to random distribution of ten trucks.

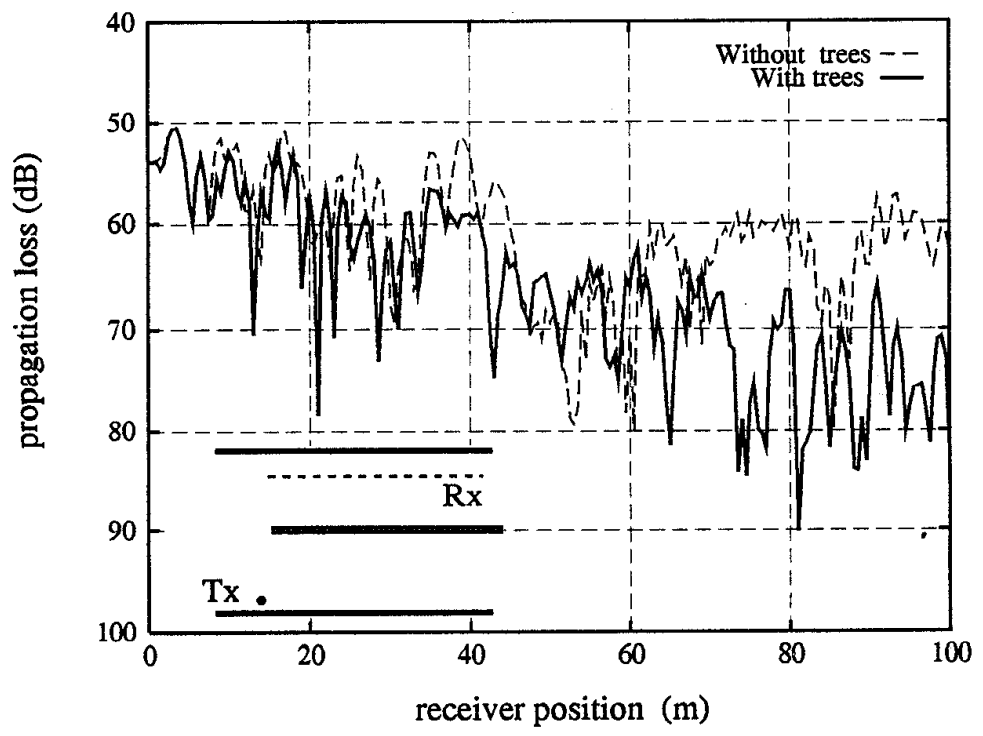

Fig. 4 Propagation loss variation according to trees. 